\title{
eHealth Literacy Plays Critical Role of Shared Decision Making: A Cross-Sectional Study
}

\author{
Chi-Chang Chang ${ }^{1,2}$, Diao $\mathrm{Ma}^{3}$, Yen-Chiao $\mathrm{Lu}^{4^{*}}$, Chalong Cheewakriangkrai ${ }^{5}$
}

\author{
${ }^{1}$ School of Medical Informatics, Chung-Shan Medical University, Taichung, Taiwan, ROC \\ 2 IT Office, Chung Shan Medical University Hospital, Taichung, Taiwan, ROC \\ 3 Department of Information Management and Information System, Wenzhou Medical University, China \\ ${ }^{4}$ School of Nursing, Chung-Shan Medical University, Taichung, Taiwan, ROC \\ ${ }^{5}$ Division of Gynecologic Oncology, Department of Obstetrics and Gynecology, Faculty of Medicine, Chiang \\ Mai University, Chiang Mai, Thailand
}

\begin{abstract}
ABSTACT
Despite the growing literature on coping strategies related variables predict health-related knowledge, yet little is known about eHealth literacy may be predicted by patient's Shared Decision Making. This study adopts a cross-sectional design to investigate whether the information ability has a mediating/moderating effects between health literacy and shared medical decision making. All participants completed the health literacy, ehealth literacy, and shared medical decision making questionnaires, respectively. The shared medical decision making index was used as an outcome measure. The mediation models and mediating hypotheses were tested by applying hierarchical multiple regression analyses. The results of this study show that female health literacy scores higher than male $(T=3.466 \quad P=0.001)$, ehealth literacy $(T=-0.362 \quad P=0.781)$ and shared medical decision making $(T=-1.555 \quad P=0.121)$; medical students' Health education manual score was significantly higher than that of non-medical students ( $T=3.265$ $P=0.001$ ). The finding suggests that the college students' ehealth literacy has fully mediating effects between health literacy and shared medical decision making. Overall, it is important for health providers to consider the notion that more ehealth literacy may sometimes, but not always, be better. Discussion highlights the need to examine nonlinear as well as linear relationships.
\end{abstract}

Table 1. Analysis of demographic variables

\begin{tabular}{|l|l|l|l|l|}
\hline coearch & use & evaluation & confidence \\
\hline risk communication & $.361^{* *}$ & $.393^{* *}$ & $.384^{* *}$ & $.389^{* *}$ \\
\hline patient autonomy & $.272^{* *}$ & $.354^{* *}$ & $.372^{* *}$ & $.315^{* *}$ \\
\hline health education essay & $.342^{* *}$ & $.357^{* *}$ & $.374^{* *}$ & $.359^{* *}$ \\
\hline outpatient dialogue & $.152^{*}$ & .119 & $.224^{* *}$ & $.162^{*}$ \\
\hline drug information & $.178^{* *}$ & $.140^{*}$ & $.245^{* *}$ & $.229^{* *}$ \\
\hline medical service system & $.308^{* *}$ & $.272^{* *}$ & $.391^{* *}$ & $.358^{* *}$ \\
\hline & $.250^{* *}$ & $.252^{* *}$ & $.319^{* *}$ & $.343^{* *}$ \\
\hline
\end{tabular}

With the Internet becoming the preferred medium for seeking health information, electronic resources are becoming more and more important in consumer health. The importance of the Internet in accessing health information has prompted people to create a large number of electronic health information resources to help consumers gain knowledge that is conducive to promoting and sustain personal health. Broadly speaking, electronic health can also be considered as an area where information and communication technology design can provide health-related information and medical information.

Themes of e-health and health literacy are closely related to public health. Health Literacy is defined in the Institute of Medicine report, it is defined as "the degree to which individuals have the capacity to obtain, process, and understand basic health information and services needed to make appropriate health decisions" [1] Based on the definition of health literacy by Norman and Skinner "underscores the importance of contextual factors that mediate health information and the need to consider health literacy in relation to the medium by which health resources are presented" [2]
With the emergence of electronic medical records, health care mobile App, and other health-related informatics technologies are increasingly capability and evaluating health information resources. For this viewpoint, e-health literacy is an important set of skills for finding effective and reliable information health professionals based on the network environment. Many studies focused on the relationship between health outcomes and literacy in the context of paper resources, not in the context of electronic resources. Therefore, electronic health literacy is still a new concept with different definitions and models. To determine consumer's e-health literacy, one should first clarify the specific knowledge, skills, abilities and other user attributes considered in previous e-health literacy studies. Health care is a process of effective communication between doctors and patients.

To meet the consumer's demand for excellence in health care, health care workers have an important role to play in preventive services and the continuation of these services is important in the health care system. Recently, patient-centered shared medical decision-making is the key factor for decision-making of effective participation. In general, a good doctor-patient relationship is a prerequisite for determining the quality of the medical.

In addition, the ineffective communication between them two important roles involved in the communication. while the increase in the self-awareness of the patient concerned as the leading role asymmetry with their owned health knowledge have resulted in the sufficient dissatisfaction of the medical process and the result. Recently, the Internet has become an important resource for people to obtain health information. The level of their ability directly affects the access of citizens to apply health knowledge to solve health problems. The health literacy formed by the health knowledge and skills acquired through e-media is called E-health literacy.

Therefore, improving the E-health literacy of college students is also the main way to improve health literacy, improve doctorpatient communication, settle down doctor-patient disputes, and promote the healthy development of doctor-patient relationship. Although the people's access to health knowledge has undergone tremendous changes, how much impact they will have on their health literacy, and how this will directly or indirectly affect the efficiency and effectiveness of doctor-patient communication, it still mystery, and the effect to share medical decision-making.

Therefore, by the statistical approach explores the relationship between health literacy and shared medical decision-making, and the mediation/regulation effect of e-health literacy between health literacy and shared medical decision making. 